\section{Febuxostat Hypersensitivity}

\section{To the Editor:}

Allopurinol hypersensitivity syndrome (AHS) is an idiosyncratic cell-mediated hypersensitivity to allopurinol ${ }^{1}$ characterized by a diffuse morbilliform rash (which can evolve into exfoliative dermatitis), eosinophilia, worsening renal function, and fever ${ }^{2}$. Historical risk factors for AHS include renal insufficiency and diuretic use ${ }^{3}$, and because the syndrome can prove fatal, physicians managing hyperuricemia prescribe allopurinol with extreme caution ${ }^{4}$, particularly in patients with renal insufficiency ${ }^{5}$. With the introduction of febuxostat to the US market in 2009, patients with a history of AHS and/or renal disease gained an option for urate-lowering therapy, and physicians could prescribe febuxostat to at-risk populations with reduced concern of AHS. A syndrome similar to AHS had not been reported in any of febuxostat's clinical trials, and none has been reported in the literature.

We describe the case of a 69 -year-old woman with multiple medical problems including gout and moderate renal insufficiency, who developed a hypersensitivity syndrome similar to AHS shortly after initiating febuxostat. She was referred to the University of Connecticut Health Center rheumatology clinic for recurrent attacks of acute gouty arthritis. She had been presumptively diagnosed with gout several years before, having had several acute arthritic episodes involving the feet and ankles, and was eventually referred to rheumatology for treatment of an acute monoarticular episode of knee pain and swelling. At the first rheumatology visit in 2005, knee arthrocentesis was performed, and under polarizing microscopy, numerous intracellular and extracellular negatively birefringent needle-shaped crystals were seen. Laboratory studies demonstrated a uric acid level of $11.2 \mathrm{mg} / \mathrm{dl}(666.2 \mu \mathrm{mol} / \mathrm{l})$ and a creatinine level of $2.0 \mathrm{mg} / \mathrm{dl}$ $(176.8 \mu \mathrm{mol} / \mathrm{l})$. After 2 weeks of colchicine monotherapy, low-dose allopurinol was added $(100 \mathrm{mg} /$ day), but was self-discontinued after a facial rash developed. The rash resolved with discontinuation of allopurinol, and the drug was not reinstituted due to concerns about allopurinol hypersensitivity.

She was lost to followup from 2006 until March 2009, when she was again referred to rheumatology after being hospitalized for polyarticular gout (wrists and knees) twice in 4 months. On presentation in the clinic, she complained of persistent discomfort in the right wrist and third metacarpophalangeal (MCP) joint. The musculoskeletal examination was notable for swelling, tenderness, and slightly decreased range of motion at the right third MCP joint. Her uric acid was $10.7 \mathrm{mg} / \mathrm{dl}(636.4 \mu \mathrm{mol} / \mathrm{l})$, and creatinine was $1.8 \mathrm{mg} / \mathrm{dl}(159.1 \mu \mathrm{mol} / 1$; estimated glomerular filtration rate $=36)$.

Other medical history included non-insulin-dependent diabetes, hypertension, gastroesophageal reflux disease, and anemia attributed to renal insufficiency. Her medications, none of which had been initiated recently, were epoetin alfa, colchicine, valsartan, glipizide, nifedipine, omeprazole, and furosemide, all at stable doses. She was taking no herbs or supplements, aside from 1 multivitamin daily.

Febuxostat was initiated at $40 \mathrm{mg}$ daily and then titrated to $80 \mathrm{mg}$ daily after 1 week. Within days of switching to the higher dose, she developed diffuse pruritus and fatigue, along with complaints that her tongue felt "raw" and appeared unusually red. She called to discuss these symptoms 2 weeks after initiating febuxostat and was told to return to the clinic immediately.

On examination, her vital signs were within normal limits, and the examination was notable for facial swelling and erythema and a markedly erythematous tongue, without other mucosal involvement. The dermatologic examination was otherwise unremarkable aside from a urticarial lesion in the left axilla. Febuxostat was discontinued. Laboratory studies revealed a creatinine of $2.8 \mathrm{mg} / \mathrm{dl}(247.5 \mu \mathrm{mol} / \mathrm{l})$, as well as a peripheral eosinophilia of $16 \%$. Interestingly, the uric acid had normalized to 5.8 $\mathrm{mg} / \mathrm{dl}(345 \mu \mathrm{mol} / \mathrm{l})$. Prednisone was prescribed at a dose of $40 \mathrm{mg}$ daily for 5 days, and laboratory data were closely monitored for the next 5 days on an outpatient basis, during which time the creatinine steadily returned to the previous baseline of $1.8 \mathrm{mg} / \mathrm{dl}(159.1 \mu \mathrm{mol} / \mathrm{l})$.

This case details the first case of febuxostat hypersensitivity reported in the literature, to my knowledge. The constellation of findings was similar to that seen in allopurinol hypersensitivity, including skin, mucosal, and renal involvement, as well as peripheral eosinophilia. Given the temporal sequence of events, as well as the spontaneous resolution of the syndrome once febuxosat was discontinued, it was concluded that febuxostat was the causative agent. Given the severe and potentially life-threatening reaction, a rechallenge was deemed inappropriate.

Based on this report, it appears that febuxostat hypersensitivity does exist, although it appears to occur much less frequently than AHS. It is unclear whether the same risks that predispose patients to AHS may predispose them to febuxostat hypersensitivity, but it is worth noting that this patient had moderate renal insufficiency and was on diuretic therapy. Additionally, it may be of importance that the patient had a history of an allopurinol-induced rash. Although a history of allopurinol hypersensitivity is not a contraindication to taking febuxostat, patients with a history of AHS were excluded from febuxostat's phase III studies. A case series of 13 patients with a history of AHS who were then placed on febuxostat reported that only 1 experienced a hypersensitivity reaction to the drug (leukocytoclastic vasculitis) ${ }^{6}$.

Febuxostat hypersensitivity appears to exist but is likely quite rare, and further data are needed to determine whether there are risk factors for its development.

ARYEH M. ABELES, MD, Assistant Clinical Professor, Division of Rheumatology, Department of Medicine, University of Connecticut Health Center, 263 Farmington Avenue, Farmington, Connecticut 06030, USA.E-mail: aabeles@uchc.edu

\section{REFERENCES}

1. Braden GL, Warzynski MJ, Golightly M, Ballow M. Cell-mediated immunity in allopurinol-induced hypersensitivity. Clin Immunol Immunopathol 1994;70:145-51.

2. Mills RM Jr. Severe hypersensitivity reactions associated with allopurinol. JAMA 1971;216:799-802.

3. Hande KR, Noone RM, Stone WJ. Severe allopurinol toxicity. Description and guidelines for prevention in patients with renal insufficiency. Am J Med 1984;76:47-56.

4. Sarawate CA, Brewer KK, Yang W, Patel PA, Schumacher HR, Saag KG, et al. Gout medication treatment patterns and adherence to standards of care from a managed care perspective. Mayo Clin Proc 2006;81:925-34.

5. Stamp LK, O’Donnell JL, Zhang M, James J, Frampton C, Barclay ML, et al. Using allopurinol above the dose based on creatinine clearance is effective and safe in patients with chronic gout, including those with renal impairment. Arthritis Rheum 2011;63:412-21.

6. Chohan S. Safety and efficacy of febuxostat treatment in subjects with gout and severe allopurinol adverse reactions. J Rheumatol 2011;38:1957-9.

J Rheumatol 2012;39:3; doi:10.3899/jrheum.111161 